\title{
THE MILNOR FIBRE OF THE PFAFFIAN AND THE HILBERT SCHEME OF FOUR POINTS ON $\mathbb{C}^{3}$
}

\author{
Alexandru Dimca and Balázs SzendröI
}

\begin{abstract}
We study a natural Hodge module on the Hilbert scheme of four points on affine three-space, which categorifies the Donaldson-Thomas invariant of the Hilbert scheme. We determine the weight filtration on the Hodge module explicitly in terms of intersection cohomology complexes, and compute the E-polynomial of its cohomology. The computations make essential use of a description of the singularity of the Hilbert scheme as the degeneracy locus of the Pfaffian function.
\end{abstract}

\section{Introduction}

Let $\left(\mathbb{C}^{3}\right)^{[m]}$ be the Hilbert scheme of $m$ points on affine three-space. By general results, $\left(\mathbb{C}^{3}\right)^{[m]}$ is known to be a separated quasi-projective scheme, equipped with a proper morphism, the Hilbert-Chow morphism, to the $m$-th symmetric product

$$
\pi_{m}:\left(\mathbb{C}^{3}\right)^{[m]} \rightarrow S^{m} \mathbb{C}^{3} .
$$

As it is well known [13], the Hilbert scheme of points of a smooth surface is itself smooth and irreducible, and $\pi_{m}$ is a resolution of singularities of the symmetric product. These statements fail for threefolds; $\left(\mathbb{C}^{3}\right)^{[m]}$ is already singular for $m=4$, and for large $m$, it is a highly reducible and non-reduced scheme [5].

On the other hand, recent work in supersymmetric gauge theory led to a description of the Hilbert scheme as a degeneracy locus. A choice of affine Calabi-Yau structure on $\mathbb{C}^{3}$ induces an embedding of $\left(\mathbb{C}^{3}\right)^{[m]}$ into a smooth quasi-projective variety $M_{m}$ of dimension $2 m^{2}+m$, which in turn is equipped with a regular function $f_{m}: M_{m} \rightarrow \mathbb{C}$, such that

$$
\left(\mathbb{C}^{3}\right)^{[m]}=\left\{d f_{m}=0\right\} \subset M_{m}
$$

is the scheme-theoretic degeneracy locus of the function $f_{m}$ on the smooth variety $M_{m}$; see Proposition 3.1.1. The reduced space $\left(\mathbb{C}^{3}\right)_{\text {red }}^{[m]}$ therefore acquires a mixed Hodge module [28]

$$
\Phi_{m}=\varphi_{f_{m}}\left(\mathbb{Q}_{M_{m}}^{H}\left[2 m^{2}+m\right]\right)\left(m^{2}-m\right) \in \operatorname{MHM}\left(\left(\mathbb{C}^{3}\right)_{\text {red }}^{[m]}\right)
$$

with underlying perverse sheaf the perverse sheaf of vanishing cycles of the function $f_{m}$; see Section 1 for the notation, and Section 3.2 for an explanation for the shift and twist appearing here.

Received by the editors April 16, 2009

2000 Mathematics Subject Classification. Primary 14C30, 14F25, 32S40; Secondary 32S55, 32S60.

Key words and phrases. Hilbert scheme, Pfaffian, Milnor fibration, vanishing cycles, intersection cohomology, mixed Hodge modules.

The first author is partially supported by ANR-08-BLAN-0317-02 (SEDIGA). The second author is partially supported by OTKA grant K61116. 
The pointwise Euler characteristic of the hypercohomology of $\Phi_{m}$ on $\left(\mathbb{C}^{3}\right)_{\text {red }}^{[m]}$ is a $\mathbb{Z}$-valued constructible function studied earlier in [1]. This constructible function can be integrated over $\left(\mathbb{C}^{3}\right)_{\text {red }}^{[\mathrm{m}]}$ to obtain an enumerative invariant, the DonaldsonThomas invariant $[32,23]$ of $\left(\mathbb{C}^{3}\right)_{\text {red }}^{[m]}$. Torus localization $[23,2]$ shows that the value of this invariant is the (signed) count of 3-dimensional partitions of weight $m$, the latter being the torus-fixed points of the Hilbert scheme under the natural torus action.

The module $\Phi_{m}$ categorifies these enumerative constructions. The purpose of this paper is to study the first non-trivial case $m=4$ in explicit topological terms. We give an alternative description of $\Phi_{4}$, using the (previously known) description of the singularities of $\left(\mathbb{C}^{3}\right)^{[4]}$ in terms of the Grassmannian $\operatorname{Gr}(2,6)$ and the Pfaffian function on skew-symmetric matrices. We use this description to compute in Theorem 3.4.1 the compactly supported E-polynomial

$$
E_{c}^{[4]}(x, y)=\sum_{p, q} \sum_{k}(-1)^{k} h^{p, q}\left(\mathbb{H}_{c}^{k}\left(\left(\mathbb{C}^{3}\right)^{[4]}, \Phi_{4}\right)\right) x^{p} y^{q},
$$

encoding the dimensions for various $p, q$ of the $(p, q)$-part of the mixed Hodge structure on the compactly supported hypercohomology $\mathbb{H}_{c}^{k}\left(\left(\mathbb{C}^{3}\right)^{[4]}, \Phi_{4}\right)$. The computation makes essential use of the standard motivic properties of the E-polynomial, recalled in Proposition 1.2.1.

The properties of $\Phi_{m}$ for general $m$, in particular the E-polynomial $E_{c}^{[m]}(x, y)$, will be studied elsewhere [3] using different methods. While the general case will present new features, the special case studied here already gives some interesting insight into the structure of these natural Hodge modules; compare Remark 3.4.2.

In Section 1, we recall general facts about mixed Hodge modules, their E-polynomials, and vanishing cycles. In Section 2, we investigate the topology of the Pfaffian singularity. In Section 3, we introduce and study the mixed Hodge module $\Phi_{4}$ on the Hilbert scheme of four points on $\mathbb{C}^{3}$.

\section{Mixed Hodge modules, E-polynomials and vanishing cycles}

1.1. Perverse sheaves and mixed Hodge modules. For a reduced and separable complex algebraic variety $V$, let $\operatorname{Perv}(V, \mathbb{Q})$ and $\operatorname{MHM}(V)$ denote respectively the abelian categories of perverse sheaves (for the middle perversity) [4] and mixed Hodge modules [28] on $V$. The realization functor

$$
\text { rat: } \operatorname{MHM}(V) \rightarrow \operatorname{Perv}(V, \mathbb{Q}),
$$

mapping a mixed Hodge module to the underlying perverse sheaf, extends to a derived functor

$$
\text { rat: } \mathcal{D}^{b} \operatorname{MHM}(V) \rightarrow \mathcal{D}_{c}^{b}(V, \mathbb{Q})
$$

to the derived category of constructible $\mathbb{Q}$-sheaves on $V$. These triangulated categories come equipped with the usual functors $f_{*}, f^{*}, f_{!}, f^{!}, \otimes, D$, compatibly under the (derived) realization functor rat.

The category of mixed Hodge modules on a point is equivalent to Deligne's category MHS of (polarizable) mixed Hodge structures, containing the canonical object $\mathbb{Q}^{H}$ of weight $(0,0)$. For a variety $V$, let $p: V \rightarrow$. be the projection to the point, then we have $\mathbb{Q}_{V}^{H}=p^{*} \mathbb{Q}^{H} \in \mathcal{D}^{b} \operatorname{MHM}(X)$; if $V$ is smooth, then in fact $\mathbb{Q}_{V}^{H}[\operatorname{dim} V] \in \operatorname{MHM}(V)$ 
is pure of weight $\operatorname{dim} V$. If $\Phi \in \mathcal{D}^{b} \operatorname{MHM}(V)$ is a complex of mixed Hodge modules, then $p_{*} \Phi, p_{!} \Phi \in$ MHS, in other words the cohomologies

$$
H^{*}\left(p_{*} \Phi\right)=\mathbb{H}^{*}(V, \Phi)
$$

and

$$
H^{*}(p ! \Phi)=\mathbb{H}_{c}^{*}(V, \Phi)
$$

carry mixed Hodge structures.

1.2. E-polynomials. Given a variety $V$ with a bounded complex $\Phi \in \mathcal{D}^{b} \operatorname{MHM}(V)$ of mixed Hodge modules, let

$$
E(V, \Phi ; x, y)=\sum_{k, p, q}(-1)^{k} h^{p, q}\left(\mathbb{H}^{k}(V, \Phi)\right) x^{p} y^{q}
$$

be its E-polynomial and

$$
E_{c}(V, \Phi ; x, y)=\sum_{k, p, q}(-1)^{k} h^{p, q}\left(\mathbb{H}_{c}^{k}(V, \Phi)\right) x^{p} y^{q}
$$

its compactly supported E-polynomial. For constant coefficients $\Phi=\mathbb{Q}_{V}^{H}$, we sometimes write $E(V, \Phi ; x, y)=E(V ; x, y)$ and $E_{c}(V, \Phi ; x, y)=E_{c}(V ; x, y)$.

Setting $x=y=1$ in $E, E_{c}$ recovers the Euler characteristic $\chi(V, \Phi)=\chi_{c}(V, \Phi)$ of (compactly supported) cohomology of $\Phi$ on $V$; see [10, Corollary 4.1.23] for this equality of Euler characteristics for any constructible sheaf complex.

If $\Phi[k]$ and $\Phi(k)$ denote the shift and the Tate twist of $\Phi$ by an integer $k$, respectively, then

$$
E(V, \Phi[k] ; x, y)=(-1)^{-k} E(V, \Phi ; x, y)
$$

and

$$
E(V, \Phi(k) ; x, y)=(x y)^{-k} E(V, \Phi ; x, y) ;
$$

the same formulae also hold for $E_{c}$. On the other hand, Verdier duality implies that if $V$ is irreducible, then

$$
E(V, \Phi ; x, y)=E_{c}\left(V, D \Phi ; x^{-1}, y^{-1}\right) .
$$

In particular, if $D(\Phi)=\Phi(n)$, then

$$
E(V, \Phi ; x, y)=x^{n} y^{n} E_{c}\left(V, D \Phi ; x^{-1}, y^{-1}\right) .
$$

By $[12,1.4]$, this applies for example to the intersection cohomology mixed Hodge module $\Phi=I C_{V}^{H}$, with $n=\operatorname{dim} V$.

Proposition 1.2.1. Let $V$ be a reduced variety and $i: Z \rightarrow V$ a closed inclusion of a reduced subvariety with complement $j: U \rightarrow V$. Given a complex of mixed Hodge modules $\Phi \in \mathcal{D}^{b} \operatorname{MHM}(V)$ on $V$, we have

$$
E_{c}(V, \Phi ; x, y)=E_{c}\left(U, j^{*} \Phi ; x, y\right)+E_{c}\left(Z, i^{*} \Phi ; x, y\right) .
$$

Proof. By $[28,2.20]$, there is an exact triangle

$$
R j_{!} j^{*} \Phi \longrightarrow \Phi \longrightarrow i_{*} L i^{*} \Phi \stackrel{[1]}{\longrightarrow}
$$

in $\mathcal{D}^{b} \operatorname{MHM}(V)$. Taking compactly supported cohomology (compact pushforward to the point) gives the result.

As a consequence, we get 
Corollary 1.2.2. Let $f: V \rightarrow T$ be a Zariski locally trivial fibration with fibre $F$. Then for constant coefficients,

$$
E_{c}(V ; x, y)=E_{c}(T ; x, y) \cdot E_{c}(F ; x, y) .
$$

Proof. This is standard; for a product, the result simply expresses the compatibility of the Künneth decomposition on compactly supported cohomology with Hodge structures. For a fibre bundle, stratify $T$ by strata over which $f$ is a product and use the previous Proposition.

1.3. Vanishing cycles. Let $f: V \rightarrow \mathbb{C}$ be a function on an $n$-dimensional smooth variety. Let ${ }^{p} \varphi_{f}\left(\mathbb{Q}_{V}[n]\right) \in \operatorname{Perv}(V, \mathbb{Q})$ be the perverse vanishing cycle sheaf [4] of $f$. By Saito's theory, this perverse sheaf underlies a canonical mixed Hodge module $\varphi_{f}\left(\mathbb{Q}_{V}^{H}[n]\right) \in \operatorname{MHM}(V)$. The support of this module is contained in the degeneracy locus

$$
Z=\{d f=0\} \subset V
$$

of $f$, so by $[28,(2.17 .5)]$, we can view $\varphi_{f}\left(\mathbb{Q}^{H}[n]\right) \in \operatorname{MHM}(Z)$.

Lemma 1.3.1. Let $V=\mathbb{C}^{n}$ be affine space, $f: V \rightarrow \mathbb{C}$ a regular function on $V$. Let $W=\mathbb{C}^{l}$ be another affine space, and $g: V \times W \rightarrow \mathbb{C}$ also regular, such that $\left.g\right|_{V \times\{0\}}=0$. Define

$$
h: V \times W \times W^{*} \rightarrow \mathbb{C}
$$

by

$$
h(v, w, \alpha)=f(v)+g(v, w)+\langle\alpha, w\rangle,
$$

where angle brackets denote the natural pairing between elements of $W$ and $W^{*}$. Then the projection $p: V \times W \times W^{*} \rightarrow V$ maps the degeneracy locus

$$
Z_{h}=\{d h=0\} \subset V \times W \times W^{*}
$$

of $h$ isomorphically to the degeneracy locus

$$
Z_{f}=\{d f=0\} \subset V
$$

of $f$. Under this isomorphism, the vanishing cycles are related by

$$
\varphi_{h} \mathbb{Q}_{V \times W \times W^{*}}^{H}[n+2 l] \cong p^{*} \varphi_{f} \mathbb{Q}_{V}^{H}[n](-l) .
$$

Proof. In coordinates $x=\left(x_{1}, \ldots x_{n}\right)$ on $V, y=\left(y_{1}, \ldots, y_{l}\right)$ on $W$ and dual coordinates $z=\left(z_{1}, \ldots, z_{l}\right)$ on $W^{*}$, we have

$$
h(x, y, z)=f(x)+g(x, y)+\sum_{j=1}^{l} y_{j} z_{j}
$$

on $\mathbb{C}^{n+2 l}$. The condition $\left.g\right|_{V \times\{0\}}=0$ implies that we may write

$$
g(x, y)=\sum_{j=1}^{l} g_{j}(x, y) y_{j}
$$

for some polynomials $g_{j}$. By making the coordinate change $x^{\prime}=x, y^{\prime}=y$ and $z_{k}^{\prime}=z_{k}+g_{k}(x, y)$ for $k=1, \ldots, l$, we may assume that $g=0$. 
The fact that $p$ is an isomorphism on $Z_{h}$ is clear, since $Z_{h}$ is contained in the subspace $V \times\{0\}$ given by the equations $y^{\prime}=z^{\prime}=0$.

The statement on vanishing cycles is also standard using a Thom-Sebastiani type result, see [30, Section 8] for the isolated singularity case and [26, Section 4] for the general case. In fact, for any $m$, the $m$-th reduced cohomology of the local Milnor fiber $F(h)_{(z, 0,0)}$ of the $h$ at a point $(z, 0,0) \in Z_{h}$ is given (as a mixed Hodge structure) by the tensor product

$$
\tilde{H}^{m}\left(F(h)_{(z, 0,0)}, \mathbb{Q}\right)=\tilde{H}^{m-2 l}\left(F(f)_{z}, \mathbb{Q}\right) \otimes H^{2 l-1}\left(F\left(A_{1}\right), \mathbb{Q}\right)
$$

where $F\left(A_{1}\right)$ is the Milnor fibre of an $A_{1}$-singularity in $\mathbb{C}^{2 l}$. It is well known that $H^{2 l-1}\left(F\left(A_{1}\right), \mathbb{Q}\right)=\mathbb{Q}(-l)$, see for instance [9, C26, p.243].

Example 1.3.2. Suppose that $V=\mathbb{C}^{n}$ and $f=\sum_{i=1}^{2 k} z_{i}^{2}$ for some $k \leq \frac{n}{2}$. The degeneracy locus is $Z=\left\{z_{1}=\ldots=z_{2 k}=0\right\} \cong \mathbb{C}^{n-2 k}$ carrying the vanishing cycle sheaf

$$
\varphi_{f}\left(\mathbb{Q}_{V}^{H}[n]\right) \cong \mathbb{Q}_{Z}^{H}[n-2 k](-k) .
$$

The shift is given by the fact that the constructible complex underlying the vanishing cycle sheaf is perverse. The Tate twist comes from the fact that the fibre of the MHM $\varphi_{f}\left(\mathbb{Q}_{V}^{H}[n]\right)$ at $z \in Z$ is given by the cohomology group $H^{2 k-1}\left(F_{z}, \mathbb{Q}\right)$, where $F_{z}$ is the Milnor fibre of an $A_{1}$-singularity in $\mathbb{C}^{2 k}$. In particular, the Tate twist $\varphi_{f}\left(\mathbb{Q}_{V}^{H}[n]\right)(k)$ is just the constant perverse sheaf $\mathbb{Q}_{Z}^{H}[\operatorname{dim} Z]$.

\section{The topology of the Pfaffian singularity}

2.1. The space of skew-symmetric matrices. Let $\operatorname{Sk}(2 n, \mathbb{C})$ be the vector space of complex skew-symmetric $2 n \times 2 n$ matrices. It is clear that

$$
\operatorname{dim} \operatorname{Sk}(2 n, \mathbb{C})=1+2+\ldots+(2 n-1)=n(2 n-1) .
$$

The general linear group $G=G \ell(2 n, \mathbb{C})$ acts on $\operatorname{Sk}(2 n, \mathbb{C})$ by $g \cdot A=g A g^{t}$, giving rise to finitely many orbits

$$
V_{2 k}=G A_{2 k},
$$

where $k=0,1, \ldots, n$ and

$$
A_{2 k}=\left(\begin{array}{ccc}
0_{k} & I_{k} & 0 \\
-I_{k} & 0_{k} & 0 \\
0 & 0 & 0
\end{array}\right)
$$

is the standard skew-symmetric $2 n \times 2 n$ matrice of rank $2 k$. Using the techniques explained in $[8$, Chapter 5], it is easy to prove the following.

Proposition 2.1.1. There are decompositions

$$
\bar{V}_{2 k}=\bigcup_{j=0}^{k} V_{2 j}
$$

with

$$
\operatorname{dim} \bar{V}_{2 k}=k(4 n-2 k-1)
$$


Example 2.1.2. The non-degenerate skew matrices, i.e. those of maximal rank $2 n$, form a Zariski open subset, in accordance with

$$
\operatorname{dim} \bar{V}_{2 n}=n(4 n-2 n-1)=n(2 n-1)=\operatorname{dim} \operatorname{Sk}(2 n, \mathbb{C}) .
$$

The next orbit is $V_{2 n-2}$, with

$$
\operatorname{dim} \bar{V}_{2 n-2}=(n-1)(2 n+1)=\operatorname{dim} \operatorname{Sk}(2 n, \mathbb{C})-1,
$$

while the next orbit has dimension $\operatorname{dim} \bar{V}_{2 n-4}=(n-2)(2 n+3)=\operatorname{dim} \operatorname{Sk}(2 n, \mathbb{C})-6$.

2.2. The Pfaffian. It is known, see for instance [18], that there is a homogeneous polynomial $\mathrm{Pf}$ of degree $n$, called the Pfaffian, in the entries $a_{i j}$ of a general matrix $A \in \operatorname{Sk}(2 n, \mathbb{C})$, such that

$$
\operatorname{det} A=\operatorname{Pf}(A)^{2} .
$$

The hypersurface $\bar{V}_{2 n-2} \subset \operatorname{Sk}(2 n, \mathbb{C})$ in the stratification of Proposition 2.1.1 is the Pfaffian hypersurface $Z=\{\mathrm{Pf}=0\}$.

As for any homogeneous polynomial, see [9, Chapter 3], there is an associated global Milnor fibration

$$
\operatorname{Pf}: \operatorname{Sk}(2 n, \mathbb{C}) \backslash Z \rightarrow \mathbb{C}^{*},
$$

with central fibre the Pfaffian hypersurface $Z$.

Example 2.2.1. For $n=2$, denote a general matrix $A \in \operatorname{Sk}(4, \mathbb{C})$ by

$$
A=\left(\begin{array}{cccc}
0 & a & b & c \\
-a & 0 & d & e \\
-b & -d & 0 & f \\
-c & -e & -f & 0
\end{array}\right)
$$

Then $\operatorname{Pf}(A)=a f-b e+c d$ has an isolated singularity at the origin of $\operatorname{Sk}(4, \mathbb{C})=\mathbb{C}^{6}$ of type $A_{1}$.

\subsection{Monodromy and cohomology.}

Proposition 2.3.1. Let $F=\mathrm{Pf}^{-1}(1)$ be the global Milnor fibre of the Milnor fibration (2.4).

(i) The Milnor fibration (2.4) is algebraically trivial, thus

$$
\operatorname{Sk}(2 n, \mathbb{C}) \backslash Z=F \times \mathbb{C}^{*} .
$$

(ii) The Milnor fibre $F$ is 4-connected. Its Betti polynomial

$$
B(F, t)=\sum_{k} b_{k}(F) t^{k}
$$

is given by

$$
B(F, t)=\left(1+t^{5}\right)\left(1+t^{9}\right) \cdots\left(1+t^{4 n-3}\right) .
$$

In particular, if we set $d=\operatorname{dim} F=2 n^{2}-n-1$, then $b_{d}(F)=1$, i.e. the top possible non-zero Betti number of the affine smooth hypersurface $F$ is equal to 1.

(iii) The E-polynomial with constant coefficients of the Milnor fibre $F$ is

$$
E(F ; x, y)=\left(1-x^{3} y^{3}\right)\left(1-x^{5} y^{5}\right) \cdots\left(1-x^{2 n-1} y^{2 n-1}\right) .
$$


Proof. We start by proving the easy statement (i). As shown for instance in [18], under the $G$-action considered in the previous section, the Pfaffian satisfies

$$
\operatorname{Pf}(g \cdot A)=\operatorname{det}(g) \operatorname{Pf}(A)
$$

for any $g \in G$ and $A \in \operatorname{Sk}(2 n, \mathbb{C})$. It follows that the mapping

$$
h: F \times \mathbb{C}^{*} \rightarrow \operatorname{Sk}(2 n, \mathbb{C}) \backslash Z
$$

given by

$$
(A, t) \mapsto g(t) \cdot A,
$$

with $g(t)$ the diagonal matrix $(t, 1, \ldots, 1)$, is an isomorphism compatible with the maps to $\mathbb{C}^{*}$, given by the second projection and $h(A, t) \mapsto \operatorname{Pf}(h(A, t))=t$.

Now we pass to statement (ii). The connectivity result follows from the fact that codim $\operatorname{Sing}(\mathrm{Pf})=6$, see $[9$, p. 76$]$; the codimension is computed using Example 2.1.2, since $\operatorname{Sing}(\mathrm{Pf})=\operatorname{Sing}(Z)=\bar{V}_{2 n-4}$.

To compute the Betti polynomial, let $M=\operatorname{Sk}(2 n, \mathbb{C}) \backslash Z$ and note that, via the $G$-action considered before, $M$ is the homogeneous space $G \ell(2 n, \mathbb{C}) / S p(2 n, \mathbb{C})$. It follows that $M$ has the homotopy type of $M_{1}=U(2 n) / Q(n)$, with $Q(n)$ the group of $n \times n$ quaternionic orthogonal matrices. By [15, Table I, p.493], the Betti polynomial of $M_{1}$ is

$$
B\left(M_{1}, t\right)=\sum_{k} b_{k}\left(M_{1}\right) t^{k}=(1+t)\left(1+t^{5}\right)\left(1+t^{9}\right) \ldots\left(1+t^{4 n-3}\right) .
$$

Using the triviality of the Milnor fibration, we get

$$
B(F, t)=B\left(M_{1}, t\right) /(1+t)=\left(1+t^{5}\right)\left(1+t^{9}\right) \ldots\left(1+t^{4 n-3}\right) .
$$

This implies in particular that $b_{5}(F)=1$, hence the connectivity claim is the best possible one.

Finally we address statement (iii) in Proposition 2.3.1. Using [11, (6.6)] and [7, Theorem (9.1.5)], we have

$$
E(G \ell(2 n, \mathbb{C}) ; x, y)=(1-x y)\left(1-x^{2} y^{2}\right) \cdots\left(1-x^{2 n} y^{2 n}\right),
$$

as well as

$$
E(S p(2 n, \mathbb{C}) ; x, y)=\left(1-x^{2} y^{2}\right)\left(1-x^{4} y^{4}\right) \cdots\left(1-x^{2 n} y^{2 n}\right),
$$

and hence, exactly as in $[11,(6.7)]$, we have

$E(M ; x, y)=E(G \ell(2 n, \mathbb{C}) / S p(2 n, \mathbb{C}) ; x, y)=(1-x y)\left(1-x^{3} y^{3}\right) \cdots\left(1-x^{2 n-1} y^{2 n-1}\right)$.

Using the triviality of the Milnor fibration, we get

$$
E(F ; x, y)=E(M ; x, y) / E\left(\mathbb{C}^{*} ; x, y\right)=\left(1-x^{3} y^{3}\right) \cdots\left(1-x^{2 n-1} y^{2 n-1}\right) .
$$

This completes the proof.

Proposition 2.3.1 shows that the Milnor fibration of the Pfaffian is very different from the Milnor fibration of a generic homogeneous polynomial of degree $n$, where the only interesting Betti number is the top one and the monodromy is non-trivial. 
Example 2.3.2. The above formulas in the case $n=3$ yield the following information on the cohomology of the Milnor fibre, where, as before, $(k)$ denotes Tate twist:

$$
H^{0}(F, \mathbb{Q})=\mathbb{Q}(0), H^{5}(F, \mathbb{Q})=\mathbb{Q}(-3), H^{9}(F, \mathbb{Q})=\mathbb{Q}(-5), H^{14}(F, \mathbb{Q})=\mathbb{Q}(-8),
$$

while the other cohomology groups of $F$ are trivial.

2.4. The perverse sheaf of vanishing cycles. We restrict in this section to the case $n=3$. Denote by

$$
X=\{d \operatorname{Pf}=0\} \subset Z=\{\operatorname{Pf}=0\} \subset \operatorname{Sk}(6, \mathbb{C})=\mathbb{C}^{15}
$$

the singular locus of the 14-dimensional Pfaffian hypersurface $Z$. It is easy to check that the equations $\{d \mathrm{Pf}=0\}$ define a reduced affine subvariety of $\mathbb{C}^{15}$ of dimension 9 . To determine this variety explicitly, identify $A \in X=\bar{V}_{2}$ with a 2 -form on $\mathbb{C}^{6}$. Such a form, having rank at most 2 , can be written as $\ell_{1} \wedge \ell_{2}$, with $\ell_{i}$ linear forms on $\mathbb{C}^{6}$. In other words, $A$ belongs to the image of the mapping

$$
\wedge^{1} \mathbb{C}^{6} \times \wedge^{1} \mathbb{C}^{6} \rightarrow \wedge^{2} \mathbb{C}^{6}=\operatorname{Sk}(6, \mathbb{C})=\mathbb{C}^{15}
$$

As is well known, see e.g. [16, p. 209], this means that $X$ is the affine cone over the complex Grassmannian $Y=\operatorname{Gr}(2,6)$ in its Plücker embedding into $\mathbb{P}^{14}$. In particular, $X$ has an isolated singularity at the vertex 0 of the cone.

Lemma 2.4.1. The variety $U=X \backslash\{0\}$ is simply-connected.

Proof. The long exact homotopy sequence of the induced Hopf fibration on $Y$

$$
\mathbb{C}^{*} \rightarrow U \rightarrow Y
$$

and the fact that $Y$ is simply-connected imply that $\pi_{1}(U)$ is a cyclic group (possibly trivial). The Thom long exact sequence of mixed Hodge structures of the fibration (2.7) reads

$$
\cdots \rightarrow H^{k}(Y, \mathbb{Z}) \rightarrow H^{k}(U, \mathbb{Z}) \rightarrow H^{k-1}(Y, \mathbb{Z})(-1) \rightarrow H^{k+1}(Y, \mathbb{Z}) \rightarrow \cdots
$$

where the last morphism $H^{k-1}(Y, \mathbb{Q})(-1) \rightarrow H^{k+1}(Y, \mathbb{Q})$ is given by the cup-product by the first Chern class of the induced Hopf bundle on $Y$. The functoriality of this long exact sequence with respect to the inclusion $l: Y \rightarrow \mathbb{P}^{14}$ and the fact that $l^{*}$ : $H^{2}\left(\mathbb{P}^{14}, \mathbb{Z}\right) \rightarrow H^{2}(Y, \mathbb{Z})$ is an isomorphism by [19], imply that $H^{1}(U, \mathbb{Z})=H^{2}(U, \mathbb{Z})=$ 0 . Finally this shows that $\pi_{1}(U)=H_{1}(U, \mathbb{Z})=0$.

Consider the perverse vanishing cycle sheaf ${ }^{p} \varphi_{\mathrm{Pf}}(\mathbb{Q}[15]) \in \operatorname{Perv}(X, \mathbb{Q})$ of the Pfaffian function. Consider also the intersection cohomology sheaf

$$
I C_{X}=j_{! *} \mathbb{Q}_{U}[9] \in \operatorname{Perv}(X, \mathbb{Q}),
$$

where $j: U \rightarrow X$ is the inclusion of the smooth part, and $j_{!_{*}}$ is the intermediate extension functor [4].

Proposition 2.4.2. Using the above notation, one has the following.

(i) On the open set $U=X \backslash\{0\}$, the two perverse sheaves have the same restriction, namely

$$
{ }^{p} \varphi_{\operatorname{Pf}}(\mathbb{Q}[15])\left|U=I C_{X}\right| U=\mathbb{Q}_{U}[9] .
$$

(ii) The non-zero stalks of the cohomology groups of ${ }^{p} \varphi_{\mathrm{Pf}}(\mathbb{Q}[15])$ at the origin are

$$
\mathcal{H}^{k}\left({ }^{p} \varphi_{\mathrm{Pf}}(\mathbb{Q}[15])\right)_{0}=\mathbb{Q} \text { for } k=0,-5,-9 .
$$


(iii) The non-zero stalks of the cohomology groups of $I C_{X}$ at the origin are

$$
\mathcal{H}^{k}\left(I C_{X}\right)_{0}=\mathbb{Q} \text { for } k=-1,-5,-9 .
$$

Proof. (i) The fact that $I C_{X} \mid U=\mathbb{Q}_{U}[9]$ is clear from (2.9). To prove the other equality, let $P \in U$ be a smooth point of $X$. Since the critical locus $X=\{d \operatorname{Pf}=0\}$ is reduced and smooth near $P$, it follows from the Morse lemma with parameters that in appropriate analytic coordinates around $P$, the Jacobian ideal of Pf is given by $J_{\mathrm{Pf}}=\left(x_{1}, \ldots, x_{c}\right)$, where $c=\operatorname{codim}(X)=6$, and in these coordinates, locally $\mathrm{Pf}=\sum_{i=1}^{c} x_{i}^{2}$. Since the vanishing cycle sheaf of this Morse function is of rank one, concentrated in the appropriate degree, it follows that there exists a rank one local system $\mathcal{L}$ on $U$ such that ${ }^{p} \varphi_{\operatorname{Pf}}(\mathbb{Q}[15])=\mathcal{L}[9]$. In view of Lemma 2.4.1, necessarily $\mathcal{L}=\mathbb{Q}_{U}$, completing the proof of (i).

Statement (ii) follows directly from Proposition 2.3.1 (ii) above, upon noting

$$
\mathcal{H}^{k}\left({ }^{p} \varphi_{\mathrm{Pf}}(\mathbb{Q}[15])\right)_{0}=\tilde{H}^{14+k}(F, \mathbb{Q}) .
$$

To prove (iii), recall that since 0 is an isolated singularity of $X$,

$$
I C_{X}=\tau_{\leq-1}\left(R j_{*} \mathbb{Q}_{U}[9]\right),
$$

see [10, Prop 5.2.10, Proof of Prop. 5.4.4]. This implies that

$$
\mathcal{H}^{k}\left(I C_{X}\right)_{0}=\mathcal{H}^{k}\left(R j_{*} \mathbb{Q}_{U}[9]\right)_{0}
$$

for $k \leq-1$ and $\mathcal{H}^{0}\left(I C_{X}\right)_{0}=0$.

On the other hand, $\mathcal{H}^{k}\left(R j_{*} \mathbb{Q}_{U}[9]\right)_{0}=H^{k+9}(U, \mathbb{Q})$ since $U$ has the same homotopy type as the link of the singularity $(X, 0)$. In the long exact sequence $(2.8)$ associated to the fibration $(2.7)$, the last morphism $H^{k-1}(Y, \mathbb{Q}) \rightarrow H^{k+1}(Y, \mathbb{Q})$ is injective for $k \leq \operatorname{dim} Y=8$ by the Hard Lefschetz Theorem, since it is given by the cup-product by the first Chern class of the induced Hopf bundle on $Y$, a non-zero multiple of the Kähler class of $Y$. This implies that, for $k \leq 9$, we have

$$
b_{k}(U)=b_{k}(Y)-b_{k-2}(Y) .
$$

The Betti polynomial of the Grassmannian $Y=\operatorname{Gr}(2,6)$ is well known:

$$
B(Y, t)=\frac{\left(1-t^{10}\right)\left(1-t^{12}\right)}{\left(1-t^{2}\right)\left(1-t^{4}\right)} .
$$

It follows that the only non-zero Betti numbers $b_{k}(U)$ for $k \leq 9$ are

$$
b_{0}(U)=b_{4}(U)=b_{8}(U)=1 .
$$

This completes the proof of (iii).

Remark 2.4.3. For future use, note that the exact sequence (2.8) of mixed Hodge structures and the fact that the cohomology groups $H^{2 k}(Y, \mathbb{Q})$ have pure Hodge type $(k, k)$ for all $k$, imply that $H^{0}(U, \mathbb{Q})=\mathbb{Q}(0), H^{4}(U, \mathbb{Q})=\mathbb{Q}(-2), H^{8}(U, \mathbb{Q})=$ $\mathbb{Q}(-4), H^{9}(U, \mathbb{Q})=\mathbb{Q}(-5), H^{13}(U, \mathbb{Q})=\mathbb{Q}(-7), H^{17}(U, \mathbb{Q})=\mathbb{Q}(-9)$.

Remark 2.4.4. Since $X$ is a cone and both sheaves ${ }^{p} \varphi_{\operatorname{Pf}}(\mathbb{Q}[15])$ and $I C_{X}$ are constructible with respect to the obvious stratification $(\{0\}, U)$ of $X$, it follows that for any $k$,

$$
\mathbb{H}^{k}\left(X,{ }^{p} \varphi_{\operatorname{Pf}}(\mathbb{Q}[15])\right)=\mathbb{H}^{k}\left(B \cap X,{ }^{p} \varphi_{\operatorname{Pf}}(\mathbb{Q}[15])\right)=\mathcal{H}^{k}\left({ }^{p} \varphi_{\operatorname{Pf}}(\mathbb{Q}[15])\right)_{0},
$$


and similarly

$$
\mathbb{H}^{k}\left(X, I C_{X}\right)=\mathbb{H}^{k}\left(B \cap X, I C_{X}\right)=\mathcal{H}^{k}\left(I C_{X}\right)_{0} .
$$

Here $B$ is a (small) ball centered at the origin, the first isomorphisms come from the conic structure of $X$ and the ones involving the stalk at 0 from [10, Corollary 4.3.11].

Denote by $i: 0 \rightarrow X$ the inclusion of the singular point.

Theorem 2.4.5. In the category $\operatorname{Perv}(X, \mathbb{Q})$ of perverse sheaves on $X$, there is a three-step filtration on ${ }^{p} \varphi_{\operatorname{Pf}}(\mathbb{Q}[15])$, with the quotients being $i_{*} \mathbb{Q}_{0}, I C_{X}$ and $i_{*} \mathbb{Q}_{0}$ respectively.

Proof. Following the arguments of [10, pp. 134-135], Theorem 2.4.2 (i) above shows that there exist a commutative diagram

$$
\begin{array}{ccccc}
{ }^{p} R j_{1} \mathbb{Q}_{U}[9] & \stackrel{\alpha}{\longrightarrow} & p \varphi_{\mathrm{Pf}}(\mathbb{Q}[15]) & \longrightarrow & { }^{p} R j_{*} \mathbb{Q}_{U}[9] \\
\| & & & & \| \\
{ }^{p} R j_{!} \mathbb{Q}_{U}[9] & \rightarrow & I C_{X} & \hookrightarrow & { }^{p} R j_{*} \mathbb{Q}_{U}[9] .
\end{array}
$$

Let $A$ denote the image of the map $\alpha$. Then we obtain the diagram

$$
\begin{aligned}
& { }^{p} R j_{1} \mathbb{Q}_{U}[9] \quad \rightarrow \quad A \quad \longrightarrow \quad{ }^{p} R j_{*} \mathbb{Q}_{U}[9] \\
& \|\quad\| \\
& { }^{p} R j_{!} \mathbb{Q}_{U}[9] \quad \rightarrow I C_{X} \quad \hookrightarrow \quad{ }^{p} R j_{*} \mathbb{Q}_{U}[9] .
\end{aligned}
$$

The definition of $A$, and an easy diagram chase in this diagram give two short exact sequences

$$
0 \rightarrow A \rightarrow{ }^{p} \varphi_{\mathrm{Pf}}(\mathbb{Q}[15]) \rightarrow Q_{1} \rightarrow 0
$$

and

$$
0 \rightarrow Q_{2} \rightarrow A \rightarrow I C_{X} \rightarrow 0
$$

in the abelian category of perverse sheaves on $X$. Again by Theorem 2.4 .2 (i), the perverse sheaves $Q_{j}$ are supported on the singular point 0 , and so they are of the form $i_{*} V_{j}$ for $\mathbb{Q}$-vector spaces $V_{j}$. Taking hypercohomology at 0 , using $\mathcal{H}^{k}\left(i_{*} V_{j}\right)_{0}=0$ for $k \neq 0$, as well as the results of Theorem 2.4.2 (ii)-(iii), we get two short exact sequences of vector spaces

$$
0 \rightarrow \mathcal{H}^{0}(A)_{0} \rightarrow \mathbb{Q} \rightarrow V_{1} \rightarrow 0,
$$

and

$$
0 \rightarrow \mathcal{H}^{-1}(A)_{0} \rightarrow \mathbb{Q} \rightarrow V_{2} \rightarrow 0 .
$$

Clearly $V_{1}=0, V_{2}=0$ are impossible, so they are both one-dimensional $\mathbb{Q}$-vector spaces. Hence the sequences (2.10)-(2.11) exhibit the claimed filtration on the vanishing cycle sheaf ${ }^{p} \varphi_{\mathrm{Pf}}(\mathbb{Q}[15])$.

Note that by [10, Prop. 4.2.10, Prop. 3.3.7, Prop. 5.2.9] respectively, the sheaves ${ }^{p} \varphi_{\mathrm{Pf}}(\mathbb{Q}[15]), I C_{X}$ and $i_{*} \mathbb{Q}_{0}$ are all self-dual under Verdier duality. This is reflected by the fact that the series of composition factors in the filtration of the sheaf of vanishing cycles is palindromic. 
2.5. The vanishing cycle Hodge module and its cohomology. In this section, we lift the results of the previous section to the category $\operatorname{MHM}(X)$ of mixed Hodge modules on $X$. By Saito's general theory, the perverse sheaf ${ }^{p} \varphi_{\operatorname{Pf}}(\mathbb{Q}[15])$ underlies a canonical mixed Hodge module $\varphi_{\mathrm{Pf}}\left(\mathbb{Q}^{H}[15]\right) \in \operatorname{MHM}(X)$.

Theorem 2.5.1. In the category $\operatorname{MHM}(X)$ of mixed Hodge modules on $X$, the weight filtration on $\varphi_{\mathrm{Pf}}\left(\mathbb{Q}^{H}[15]\right)$ has quotients $i_{*} \mathbb{Q}_{0}^{H}(-7)$ in weight $14, I C_{X}^{H}(-3)$ in weight 15 and $i_{*} \mathbb{Q}_{0}^{H}(-8)$ in weight 16 respectively. Here $I C_{X}^{H}$ and $\mathbb{Q}_{0}^{H}$ are the natural mixed Hodge modules over the perverse sheaves $I C_{X}$ and $\mathbb{Q}_{0}$ on $X$ and 0 , respectively.

Proof. The graded quotients of the weight filtration on $\varphi_{\operatorname{Pf}}\left(\mathbb{Q}^{H}[15]\right)$ are pure Hodge modules, which in turn decompose into a direct sum of objects with strict support. Hence the filtration found in Theorem 2.4.5 is necessarily the filtration by perverse sheaves underlying the weight filtration. The proof of Theorem 2.4.5 also shows that the graded pieces are as claimed, once we replace the isomorphism

$$
{ }^{p} \varphi_{\mathrm{Pf}}(\mathbb{Q}[15])\left|U=I C_{X}\right| U=\mathbb{Q}_{U}[9]
$$

from Proposition 2.4.2 by the mixed Hodge module isomorphism

$$
\varphi_{\mathrm{Pf}}\left(\mathbb{Q}^{H}[15]\right)\left|U=I C_{X}(-3)\right| U=\mathbb{Q}_{U}^{H}(-3)[9] .
$$

For the Tate twist, see Example 1.3.2.

Next, one may use the exact sequence (2.11) and Remark 2.4.3 to identify

$$
Q_{2}=\mathcal{H}^{-1}\left(I C_{X}\right)_{0}(-3)=H^{8}(U, \mathbb{Q})(-3)=\mathbb{Q}(-7) .
$$

On the other hand, the exact sequence (2.10) gives

$$
Q_{1}=\mathcal{H}^{0}\left({ }^{p} \varphi_{\mathrm{Pf}}\left(\mathbb{Q}^{H}[15]\right)\right)=H^{14}(F, \mathbb{Q})=\mathbb{Q}(-8)
$$

according to Example 2.3.2.

Finally, it is well known that for any irreducible variety $V$, the intersection complex $I C_{V}^{H}$ is pure of weight $n=\operatorname{dim} V$, see for instance [25, Lemma 14.15].

Theorem 2.5.2. The E-polynomials of the mixed Hodge Module $\left.\varphi_{\mathrm{Pf}}\left(\mathbb{Q}^{H}[15]\right)\right)$ are given by

and

$$
E\left(X, \varphi_{\mathrm{Pf}}\left(\mathbb{Q}^{H}[15]\right) ; x, y\right)=(x y)^{3}\left((x y)^{5}-(x y)^{2}-1\right)
$$

$$
E_{c}\left(X, \varphi_{\operatorname{Pf}}\left(\mathbb{Q}^{H}[15]\right) ; x, y\right)=(x y)^{7}\left(1-(x y)^{3}-(x y)^{5}\right) .
$$

Proof. Using Remark 2.4.4 we get the following isomorphisms of MHS

$$
\mathbb{H}^{k}\left(X, \varphi_{\operatorname{Pf}}\left(\mathbb{Q}^{H}[15]\right)\right)=\mathcal{H}^{k}\left(\varphi_{\operatorname{Pf}}\left(\mathbb{Q}^{H}[15]\right)\right)_{0}=\tilde{H}^{14+k}(F, \mathbb{Q}) .
$$

The formula for $E\left(X, \varphi_{\operatorname{Pf}}\left(\mathbb{Q}^{H}[15]\right) ; x, y\right)$ follows then from Example 2.3.2. To compute the E-polynomial with compact supports, note that $\varphi_{\operatorname{Pf}}\left(\mathbb{Q}^{H}[15]\right)$ contains as a direct summand $\varphi_{\mathrm{Pf}, 1}\left(\mathbb{Q}^{H}[15]\right)$, on which the semisimple part of the monodromy acts trivially. Since the underlying perverse sheaf ${ }^{p} \varphi_{\mathrm{Pf}}(\mathbb{Q}[15])$ admits no non-trivial direct summand, we must have

$$
\varphi_{\mathrm{Pf}, 1}\left(\mathbb{Q}^{H}[15]\right)=\varphi_{\mathrm{Pf}}\left(\mathbb{Q}^{H}[15]\right) .
$$

Now using $[28,(2.6 .2)]$ and the identification $I C_{\mathbb{C}^{15}}^{H}=\mathbb{Q}^{H}[15]$, we get

$$
D\left(\varphi_{\operatorname{Pf}}\left(\mathbb{Q}^{H}[15]\right)\right)=\varphi_{\operatorname{Pf}}\left(D\left(\mathbb{Q}^{H}[15]\right)\right)=\varphi_{\operatorname{Pf}}\left(\mathbb{Q}^{H}[15]\right)(15) .
$$


Hence (1.2) applies.

\section{The Hilbert scheme of four points on affine three-space}

3.1. The superpotential description. Let $T$ be the three-dimensional space of linear functions on $\mathbb{C}^{3}$, so that $\mathbb{C}^{3}=\operatorname{Spec} \operatorname{Sym}{ }^{\bullet} T$. Fix an isomorphism vol : $\wedge^{3} T \cong \mathbb{C}$; this corresponds to choosing a holomorphic volume form (Calabi-Yau form) on $\mathbb{C}^{3}$. We start by recalling the description of the Hilbert scheme as a degeneracy locus from [31, Proof of Thm. 1.3.1] and [29, Prop.3.8].

Proposition 3.1.1. The pair ( $T$, vol) defines an embedding of the Hilbert scheme $\left(\mathbb{C}^{3}\right)^{[m]}$ into a smooth quasi-projective variety $M_{m}$, which in turn is equipped with a regular function $f_{m}: M_{m} \rightarrow \mathbb{C}$, such that

$$
\left(\mathbb{C}^{3}\right)^{[m]}=\left\{d f_{m}=0\right\} \subset M_{m}
$$

is the scheme-theoretic degeneracy locus of the function $f_{m}$ on $M_{m}$.

Proof. A point $[Z] \in\left(\mathbb{C}^{3}\right)^{[m]}$ corresponds to an embedded 0-dimensional subscheme $Z \hookrightarrow \mathbb{C}^{3}$ of length $m$, in other words to a quotient $\mathcal{O}_{\mathbb{C}^{3}} \rightarrow \mathcal{O}_{Z}$ with $H^{0}\left(\mathcal{O}_{Z}\right)$ of dimension $m$. Fixing an $m$-dimensional complex vector space $W_{m}$, the data defining a cluster consists of a linear map $\phi: T \otimes W_{m} \rightarrow W_{m}$, subject to the condition that the induced action of the tensor algebra of $T$ factors through an action of the symmetric algebra $\operatorname{Sym}^{\bullet} T$, and a vector $1 \in W_{m}$ which generates $W_{m}$ under the action.

Let

$$
U_{m} \subset \operatorname{Hom}\left(T \otimes W_{m}, W_{m}\right) \times W_{m}
$$

denote the space of maps with cyclic vector, but without the symmetry condition. As proved in [31, Lemma 1.2.1], the action of $G \ell\left(W_{m}\right)$ on $U_{m}$ is free, and the quotient

$$
M_{m}=U_{m} / G \ell\left(W_{m}\right)
$$

is a smooth quasiprojective GIT quotient.

Finally consider the map

$$
\phi \mapsto \operatorname{Tr}\left(\wedge^{3} \phi\right),
$$

where $\wedge^{3} \phi: \bigwedge^{3} T \times W_{m} \rightarrow W_{m}$ and we use the isomorphism vol before taking the trace on $W_{m}$. It is clear that this map descends to a regular map $f_{m}: M_{m} \rightarrow \mathbb{C}$. As proved in [29, Prop.3.8], the equations $\left\{d f_{m}=0\right\}$ are just the the equations which say that the action factors through the symmetric algebra. As proved by [24] (in dimension 2 , but the proof generalizes), the scheme cut out by these equations is precisely the moduli scheme representing the functor of $m$ points on $\mathbb{C}^{3}$. Thus, as a scheme,

$$
\left(\mathbb{C}^{3}\right)^{[m]}=\left\{d f_{m}=0\right\} \subset M_{m}
$$

Remark 3.1.2. In explicit terms, fixing a basis of $V, \mathbb{C}[x, y, z]$ acts on $W_{m}$ by a triple of commuting matrices $X, Y, Z$. The map $f_{m}$ on triples of matrices is given by

$$
(X, Y, Z) \mapsto \operatorname{Tr}[X, Y] Z .
$$


3.2. The Hodge module $\Phi_{m}$ on the Hilbert scheme. As a consequence of Proposition 3.1.1, the reduced space $\left(\mathbb{C}^{3}\right)_{\text {red }}^{[\mathrm{m}]}$ acquires a mixed Hodge module

$$
\Phi_{m}=\varphi_{f_{m}}\left(\mathbb{Q}^{H}\left[\operatorname{dim} M_{m}\right]\right)\left(m^{2}-m\right),
$$

with underlying perverse sheaf the perverse sheaf of vanishing cycles of the function $f_{m}$. By $[1,1.2]$, the pointwise Euler characteristic of $\Phi_{m}$ is the Behrend function of $\left(\mathbb{C}^{3}\right)^{[\mathrm{m}]}$, and hence the Euler characteristic over the whole space computes its Donaldson-Thomas invariant [23]. In this sense, this Hodge module categorifies the Donaldson-Thomas invariant of the Hilbert scheme.

The shift and Tate twist appearing in the definition of $\Phi_{m}$ are not essential, and are only inserted for cosmetic purposes. The shift by $\operatorname{dim} M_{m}$ simply means that the underlying constructible complex is a perverse sheaf; thus, it puts the object in the abelian category $\operatorname{MHM}\left(\left(\mathbb{C}^{3}\right)_{\text {red }}^{[m]}\right)$ and not just in its derived category. The Tate twist cancels certain Tate twists arising from the embedding in $M_{n}$. To illustrate this, note that for $m \leq 3$, the Hilbert scheme $\left(\mathbb{C}^{3}\right)^{[m]}$ is nonsingular. It is moreover easy to see, using the argument in the proof of Proposition 2.4.2(i), that the Hodge module $\Phi_{m}$ is the trivial sheaf $\mathbb{Q}^{H}\left[\operatorname{dim}\left(\mathbb{C}^{3}\right)^{[m]}\right]$ in these cases; the Tate twist in the definition of $\Phi_{m}$ cancels the Tate twist arising from Example 1.3.2. See Remark 3.4.2 for further discussion of this point. The paper [3] computes the cohomology of all the Hodge modules $\Phi_{m}$, making contact with the refined topological vertex calculations of [17]; the Tate twist will be important there to get a closed formula.

Recent work of Kontsevich and Soibelman [22] constructs a motivic generalization of Donaldson-Thomas theory in great generality, using local data. So their point of view is slightly different; the Hodge module $\Phi_{m}$ exist globally on the moduli space $\left(\mathbb{C}^{3}\right)^{[m]}$. It seems to be an interesting question when does there exist a global mixed Hodge module on a moduli space $\mathcal{M}$ admitting a symmetric perfect obstruction theory [2], whose pointwise Euler characteristic is the Behrend function of $\mathcal{M}$, and if so, then how canonical is it.

3.3. The Hodge module $\Phi_{4}$ on the Hilbert scheme of four points. For the rest of the paper, consider the case $m=4$ of four points on affine 3-space. By [21], the scheme $\left(\mathbb{C}^{3}\right)^{[4]}$ is irreducible of dimension 12 , singular along the locus $S_{4}$ of lengthfour subschemes of $\mathbb{C}^{3}$ given by the squares of maximal ideals of points. Let $N_{4}=$ $\left(\mathbb{C}^{3}\right)^{[4]} \backslash S_{4}$ be the nonsingular part.

Fix an affine structure on $\mathbb{C}^{3}$ once again, then there is a stratification

$$
L_{4} \subset P_{4} \subset\left(\mathbb{C}^{3}\right)^{[4]}
$$

defined as follows: $P_{4}$ is the locus of subschemes scheme-theoretically contained in a linear hyperplane in $\mathbb{C}^{3} ; L_{4}$ is the locus of subschemes scheme-theoretically contained in a line. The complement

$$
V_{4}=\left(\mathbb{C}^{3}\right)^{[4]} \backslash P_{4}
$$

is the open subset of all colength-four ideals $I \triangleleft \mathrm{Sym}^{\bullet} T$ such that as a vector space, $\mathrm{Sym}^{\bullet} T / I \cong \mathbf{1} \oplus T$, where $\mathbf{1}$ is the one-dimensional space generated by the cyclic vector 1 . This open subset $V_{4}$ contains the singular locus $S_{4}$ of $\left(\mathbb{C}^{3}\right)^{[4]}$. 
Proposition 3.3.1. (i) (compare $[21,20])$ The open subset $V_{4}$ of $\left(\mathbb{C}^{3}\right)^{[4]}$ is affine. There is a product decomposition $V_{4}=\mathbb{C}^{3} \times X$, where $X \subset \mathbb{C}^{15}$ is the cone over $\operatorname{Gr}(2,6)$ in its Plücker embedding in $\mathbb{P}^{14}$.

(ii) The restriction of $\Phi_{4}$ to $V_{4}$ is given by $p_{2}^{*}\left(\varphi_{\mathrm{Pf}}\left(\mathbb{Q}^{H}[15]\right)\right)[3](3)$, where $p_{2}: V_{4} \rightarrow X$ is the second projection.

(iii) The restriction of $\Phi_{4}$ to the nonsingular part $N_{4}=\left(\mathbb{C}^{3}\right)^{[4]} \backslash S_{4}$ is the rank-one sheaf $\mathbb{Q}_{N}^{H}[12]$.

Proof. First we prove (i) and (ii) together. As remarked above, on the open set $V_{4} \subset\left(\mathbb{C}^{3}\right)^{[4]}$, there is a decomposition $W_{4} \cong \mathbf{1} \oplus T$. Unwinding the definitions of the map $\phi$ from Proposition 3.1.1, we can then write

$$
\phi=\left(\begin{array}{cc}
0 & \phi_{1} \\
\operatorname{id}_{T} & \phi_{2}
\end{array}\right): T \otimes(\mathbf{1} \oplus T) \rightarrow \mathbf{1} \oplus T
$$

so the data is equivalent to a pair of maps

$$
\left(\phi_{1}, \phi_{2}\right): T \otimes T \rightarrow \mathbf{1} \oplus T
$$

leading to an embedding

$$
V_{4} \subset \operatorname{Hom}(T \otimes T, \mathbf{1} \oplus T) .
$$

Note there is no $G \ell$-action left here, since we completely rigidified $W_{4}$ using $T$. This is the restriction of the embedding (3.1), mapping the open set $V_{4} \subset\left(\mathbb{C}^{3}\right)^{[4]}$ into a 36-dimensional affine space. Thus $V_{4}$ is indeed affine.

Write $\phi_{2}=\phi_{2}^{+}+\phi_{2}^{-}$, where $\phi_{2}^{+} \in \operatorname{Hom}\left(\operatorname{Sym}^{2} T, T\right)$ and $\phi_{2}^{-} \in \operatorname{Hom}\left(\bigwedge^{2} T, T\right)$. Then a short computation shows

$$
\operatorname{Tr}\left(\wedge^{3} \phi\right)=\operatorname{Tr}\left(\wedge^{3} \phi_{2}^{+}\right)+g\left(\phi_{2}^{+}, \phi_{2}^{-}\right)+2\left\langle\phi_{1}, \phi_{2}^{-}\right\rangle .
$$

Here $g$ is some cubic function, which we will not need explicitly, and the angle brackets denote the natural pairing between $\operatorname{Hom}(T \otimes T, \mathbf{1})$ and

$$
\operatorname{Hom}\left(\bigwedge^{2} T, T\right) \cong \operatorname{Hom}\left(T^{*}, T\right) \cong T \otimes T,
$$

where the first isomorphism is induced by vol. The conditions of Lemma $1.3 .1 \mathrm{can}$ be checked to hold; so by that Lemma, the vanishing cycle sheaf of $f_{4}$ on $V_{4}$ can be computed from the embedding

$$
V_{4} \subset \operatorname{Hom}\left(\operatorname{Sym}^{2} T, T\right)
$$

as the degenerecy locus of the function $\operatorname{Tr}\left(\wedge^{3} \phi_{2}^{+}\right)$.

Next, Lemma 3.3.3 below, applied to $S=T^{*}$, together with the trivialization $\bigwedge^{3} T^{*} \cong \mathbf{1}^{*} \cong \mathbf{1}$ induced by vol: $\bigwedge^{3} T \cong \mathbf{1}$, gives an isomorphism

$$
\operatorname{Hom}\left(\operatorname{Sym}^{2} T, T\right) \cong \mathbb{C}^{3} \times \bigwedge^{2} \operatorname{Sym}^{2} T^{*}
$$

It can be checked by explicit calculation that under this isomorphism, the function $\operatorname{Tr}\left(\wedge^{3} \phi_{2}^{+}\right)$only depends on the projection to the second factor, leading to an isomorphism

$$
V_{4} \cong \mathbb{C}^{3} \times X \subset \mathbb{C}^{3} \times \bigwedge^{2} \operatorname{Sym}^{2} T^{*}
$$


and the function on $\bigwedge^{2} \mathrm{Sym}^{2} T^{*}$ is the Pfaffian of Section 2.2. This proves (i). As for the vanishing cycle sheaf, we finally have

$$
\left.\Phi_{4}\right|_{V_{4}} \cong p_{2}^{*}\left(\varphi_{\mathrm{Pf}}\left(\mathbb{Q}^{H}[15]\right)\right)[3](3),
$$

where $p_{2}: V_{4}=\mathbb{C}^{3} \times X \rightarrow X$ is the second projection. Here the additional shift [3] is due to the fact that the relative dimension of $p_{2}$ is 3 ; the Tate twist $3=12-9$ arises from the Tate twist in the definition of $\Phi_{4}$, and the reduction from the 36dimensional embedding space $\left(\mathbb{C}^{3}\right)^{[4]} \subset M_{4}$ to the 18-dimensional embedding space $\operatorname{Hom}\left(\operatorname{Sym}^{2} T, T\right)$ using Lemma 1.3.1. This concludes the proof of (ii).

To prove (iii), first of all note that the nonsingular part $\left(\mathbb{C}^{3}\right)^{[4]} \backslash S_{4}$ has a Zariski open subset $V_{4} \backslash S_{4}$ which is isomorphic by (i) to $U \times \mathbb{C}^{3}$, where $U$ is the nonsingular part of $X$. Since $U$ is simply connected by Lemma 2.4.1, so is $N_{4}$. Thus, the restriction of $\Phi_{4}$ to $N_{4}=\left(\mathbb{C}^{3}\right)^{[4]} \backslash S_{4}$ is $\mathbb{Q}_{N_{4}}^{H}[12]$, since the transversal singularity is $A_{1}$ embedded in $\mathbb{C}^{24}$, exactly as in the proof of Theorem 2.5.1; the Tate twists by 12 in the definition of $\Phi_{4}$ and -12 computed in Example 1.3.2 cancel.

Corollary 3.3.2. In the category $\operatorname{MHM}\left(\left(\mathbb{C}^{3}\right)^{[4]}\right)$ of mixed Hodge modules on $\left(\mathbb{C}^{3}\right)^{[4]}$, the weight filtration on $\Phi_{4}$ has quotients $j_{*} \mathbb{Q}_{S_{4}}^{H}[3](-4)$ in weight $11, I C_{\left(\mathbb{C}^{3}\right)^{[4]}}^{H}$ in weight 12 and $j_{*} \mathbb{Q}_{S_{4}}^{H}[3](-5)$ in weight 13 respectively, where $j: S_{4} \cong \mathbb{C}^{3} \hookrightarrow\left(\mathbb{C}^{3}\right)^{[4]}$ denotes the inclusion of the singular locus.

Proof. This follows from Proposition 3.3.1, in light of Theorem 2.5.1, by applying the functor $p_{2}^{*}(-)[3](3)$ to all the objects in Theorem 2.5.1. Note that

$$
p_{2}^{*}\left(I C_{X}^{H}\right)[3] \cong I C_{V_{4}}^{H}
$$

since both mixed Hodge modules here are pure of weight 12, it is enough to check that they are isomorphic on the perverse sheaf level, where it follows for instance using [10, Thm. 3.2.13 (ii), Thm. 3.2.17 (iii) and Prop. 5.2.10].

The proof of Proposition 3.3.1 used the following

Lemma 3.3.3. (compare [21, Lemma 1.6]) For a three-dimensional vector space $S$, there is a canonical isomorphism

$$
\operatorname{Hom}\left(\operatorname{Sym}^{2} S, S\right) \cong S^{*} \oplus \bigwedge^{2} \operatorname{Sym}^{2} S^{*} \otimes \bigwedge^{3} S .
$$

Proof. For any vector space $S$, there are canonical inclusions

$$
\begin{aligned}
S^{*} & \rightarrow \operatorname{Hom}\left(\operatorname{Sym}^{2} S, S\right) \\
\alpha & \mapsto\left(\left(s_{1}, s_{2}\right) \mapsto \alpha\left(s_{1}\right) s_{2}+\alpha\left(s_{2}\right) s_{1}\right)
\end{aligned}
$$

and

$$
\begin{aligned}
& \bigwedge^{2} \operatorname{Sym}^{2} S^{*} \otimes \bigwedge^{3} S \rightarrow \operatorname{Hom}\left(\operatorname{Sym}^{2} S, S\right) \\
& \left(\omega_{1} \wedge \omega_{2}\right) \otimes\left(s_{1} \wedge s_{2} \wedge s_{3}\right) \mapsto\left(\left(t_{1}, t_{2}\right) \mapsto \sum_{\sigma \in S_{3}}(-1)^{\operatorname{sign}(\sigma)} \omega_{1}\left(t_{1} s_{\sigma(1)}\right) \omega_{2}\left(t_{2} s_{\sigma(2)}\right) s_{\sigma(3)}\right)
\end{aligned}
$$


where $t_{i}, s_{i} \in S, \omega_{i} \in \mathrm{Sym}^{2} S^{*}$. Writing these maps out on a basis, one checks that the images intersect trivially. For $\operatorname{dim} S=3$, the Lemma follows by a dimension count.

3.4. The E-polynomial of $\Phi_{4}$ on the Hilbert scheme of four points. As in the Introduction, let

$$
E_{c}^{[4]}(x, y)=E_{c}\left(\left(\mathbb{C}^{3}\right)^{[4]}, \Phi_{4} ; x, y\right)
$$

be the E-polynomial of compactly supported cohomology of the Hodge module $\Phi_{4}$ on $\left(\mathbb{C}^{3}\right)^{[4]}$.

Theorem 3.4.1. We have

$$
E_{c}^{[4]}(x, y)=(x y)^{6}\left((x y)^{6}+(x y)^{5}+3(x y)^{4}+3(x y)^{3}+3(x y)^{2}+(x y)+1\right) .
$$

Proof. The computation makes use of the motivic nature of the compactly supported E-polynomial. We use the stratification $L_{4} \subset P_{4} \subset\left(\mathbb{C}^{3}\right)^{[4]}$ from Section 3.3.

The contribution from the open stratum $V_{4}=\left(\mathbb{C}^{3}\right)^{[4]} \backslash P_{4}$ is

$$
\begin{aligned}
E_{c}\left(V_{4},\left.\Phi_{4}\right|_{V_{4}} ; x, y\right) & \left.=E_{c}\left(V_{4}, p_{2}^{*}\left(\varphi_{\operatorname{Pf}}(\mathbb{Q}[15])\right)[3](3)\right) ; x, y\right) \\
& =E\left(V_{4}, D\left(p_{2}^{*}\left(\varphi_{\operatorname{Pf}}(\mathbb{Q}[15])\right)[3](3)\right) ; x^{-1}, y^{-1}\right) \\
& =E\left(V_{4}, p_{2}^{!}\left(\varphi_{\operatorname{Pf}}(\mathbb{Q}[15])(15)\right)[-3](-3) ; x^{-1}, y^{-1}\right) \\
& =-E\left(V_{4}, p_{2}^{*}\left(\varphi_{\operatorname{Pf}}(\mathbb{Q}[15])(15)\right) ; x^{-1}, y^{-1}\right) \\
& =-E\left(X, \varphi_{\mathrm{Pf}}(\mathbb{Q}[15])(15) ; x^{-1}, y^{-1}\right) \\
& =-E_{c}\left(X, D\left(\varphi_{\mathrm{Pf}}(\mathbb{Q}[15])(15)\right) ; x, y\right) \\
& =-E_{c}\left(X, \varphi_{\mathrm{Pf}}(\mathbb{Q}[15]) ; x, y\right) \\
& =(x y)^{7}\left((x y)^{5}+(x y)^{3}-1\right) .
\end{aligned}
$$

Here, apart from standard properties of pullback and duality, we used Proposition 3.3.1(ii) for the first equality, the identity $p_{2}^{!}=p_{2}^{*}(3)[6]$ from $[27,(3.5 .1)$ and (3.5.2)] for the fourth, and Theorem 2.5.2 for the last one.

Next, look at the smallest stratum $L_{4}$ of clusters lying in a line. By Proposition 3.3.1(iii), the restriction of $\Phi_{4}$ to this stratum is $\mathbb{Q}_{L_{4}}^{H}[12]$. Associating to a cluster in $L_{4}$ the line it is contained in defines a map $l: L_{4} \rightarrow \operatorname{Lines}\left(\mathbb{C}^{3}\right)$ to the space Lines $\left(\mathbb{C}^{3}\right)$ of all lines in $\mathbb{C}^{3}$. The map $l$ is easily seen to be a Zariski locally trivial fibration, with fibre the Hilbert scheme of four points on the affine line. The space Lines $\left(\mathbb{C}^{3}\right)$ of all lines in $\mathbb{C}^{3}$ further fibres over $\mathbb{P}^{2}$, with fibre $\mathbb{C}^{2}$. The Hilbert scheme of four points on the affine line is simply $\mathbb{C}^{4}$. Putting all this together, using Corollary 1.2.2 repeatedly, we get, for constant coefficients,

$$
E_{c}\left(L_{4} ; x, y\right)=(x y)^{4}(x y)^{2}\left(1+x y+(x y)^{2}\right) .
$$

Thus the contribution from $L_{4}$ to $E_{c}^{[4]}(x, y)$ is

$$
E_{c}\left(L_{4},\left.\Phi_{4}\right|_{L_{4}} ; x, y\right)=(x y)^{6}\left(1+x y+(x y)^{2}\right) .
$$

Finally, look at the stratum $P_{4} \backslash L_{4}$ of strictly planar clusters. By Proposition 3.3.1(iii) again, the restriction of $\Phi_{4}$ to this stratum is still a shift of the trivial sheaf $\mathbb{Q}_{P_{4} \backslash L_{4}}^{H}$. The variety $P_{4} \backslash L_{4}$ has a Zariski locally trivial fibration over the space Planes $\left(\mathbb{C}^{3}\right)$ of all planes in $\mathbb{C}^{3}$, with fibre the Hilbert scheme of four points on the affine plane which are not collinear. The space Planes $\left(\mathbb{C}^{3}\right)$ of all planes in $\mathbb{C}^{3}$ further 
fibres over $\mathbb{P}^{2}$, with fibre $\mathbb{C}$. On the other hand, the E-polynomial with constant coefficients of the Hilbert scheme of four points on the affine plane can be computed from Göttsche's formula [14] to be

$$
E\left(\left(\mathbb{C}^{2}\right)^{[4]} ; x, y\right)=(x y)^{5}+2(x y)^{6}+(x y)^{7}+(x y)^{8} .
$$

We need to subtract from this the contribution from the locus of clusters on a plane contained in a line, which can be computed by fibering it over the space of all lines in a plane. Putting all this together, we obtain

$$
\begin{aligned}
E_{c}\left(P_{4} \backslash L_{4} ; x, y\right)= & \left((x y)^{5}+2(x y)^{6}+(x y)^{7}+(x y)^{8}-(x y)^{4} x y(1+x y)\right) . \\
& \cdot x y \cdot\left(1+x y+(x y)^{2}\right) .
\end{aligned}
$$

Collecting terms, the contribution from $P_{4} \backslash L_{4}$ to $E_{c}^{[4]}(x, y)$ is

$$
E_{c}\left(P_{4} \backslash L_{4},\left.\Phi_{4}\right|_{P_{4} \backslash L_{4}} ; x, y\right)=(x y)^{7}\left(1+x y+(x y)^{2}\right)^{2} .
$$

Summing the three contributions gives the answer stated in the Theorem.

Remark 3.4.2. For $x=y=1$, we get $E_{c}^{[4]}(1,1)=13$, which is indeed the DonaldsonThomas invariant of the Hilbert scheme of four points on three-space [23, 2]. As discussed in the Introduction, the latter is the number of 3-dimensional partitions of 4, the number of torus-fixed points on the Hilbert scheme. The formula of Theorem 3.4.1 refines this enumerative count to a polynomial.

As a result of the Tate twist in the definition of $\Phi_{m}$, the expression for $E_{c}^{[4]}$ looks like the E-polynomial of a smooth variety of dimension 12. Being a sum of positive powers of $x y$, the formula could in fact correspond to a Hodge structure which is an effective sum of Tate motives. This indeed happens in the much simpler cases $m \leq 3$ : the Hilbert scheme $\left(\mathbb{C}^{3}\right)^{[m]}$ in these cases is smooth, the module $\Phi_{m}$ is simply the constant module, and the Hilbert scheme itself is just a union of pieces each of which is affine space of some dimension. The E-polynomials in these cases are computed in [6]. However, the proof shows that for $m=4$, this cannot be true, and one cannot recover the cohomology from the E-polynomial, because of cancellation between terms in different degrees.

A different way to compute the E-polynomial $E_{c}^{[4]}(x, y)$ would be by torus localization, as in the proof of Göttsche's formula for $\mathbb{C}^{2}$ given in [24, 5.2], the Euler characteristic computation of [2] on the Hilbert scheme of $\mathbb{C}^{3}$, and the computation of the Poincaré polynomial of the resolution of singularities of $\left(\mathbb{P}^{3}\right)^{[4]}$ in $[21]$. As an ideal scenario, one could have hoped in fact that each of the 13 torus-fixed points contributes a monomial of $x y$ to the E-polynomial. But this turns out to be false. Of the 13 torus-fixed points corresponding to 3 -dimensional partitions of 4 , all but one are planar and therefore lie in the smooth locus of the Hilbert scheme. The contribution of these points to the E-polynomial is easily computed using formulae from [23], a computer, and an appropriate one-dimensional subtorus of $\left(\mathbb{C}^{*}\right)^{3}$. The result is that the smooth torus-fixed points contribute

$$
(x y)^{12}+2(x y)^{11}+3(x y)^{10}+(x y)^{9}+3(x y)^{8}+(x y)^{7}+(x y)^{6} .
$$

Comparing this with the final formula, we see that the last remaining fixed point, the square of the maximal ideal at the origin which lies in the singular locus, must contribute $2(x y)^{9}-(x y)^{11}$. 


\section{Acknowledgements}

The authors would like to thank Barbara Fantechi and Lothar Göttsche for pointing out Proposition 3.3.1(i), as well as Tom Bridgeland, Patrick Brosnan, Stefan Papadima, Morihiko Saito and Duco van Straten for helpful remarks and correspondence. The first named author is grateful to ASSMS, Government College University, Lahore, Pakistan, where part of the work on this paper was done.

\section{References}

[1] K. Behrend, Donaldson-Thomas invariants via microlocal geometry, arXiv:math/0507523.

[2] K. Behrend and B. Fantechi, Symmetric obstruction theories and Hilbert schemes of points on threefolds, Algebra Number Theory 2 (2008) 313-345.

[3] K. Behrend, J. Bryan and B. Szendrői, Motivic degree zero Donaldson-Thomas invariants, arXiv:0909.5088.

[4] A. Beilinson, J. Bernstein and P. Deligne, Faisceaux pervers, in: Analysis and topology on singular spaces I, Luminy, 1981, 5-171, Astrisque 100, Soc. Math. France, Paris, 1982.

[5] J. Briançon and A. Iarrobino, Dimension of the punctual Hilbert scheme, J. Algebra 55 (1978), $536-544$.

[6] J. Cheah, On the cohomology of Hilbert schemes of points, J. Alg. Geom. 5 (1996) 479-511.

[7] P. Deligne, Théorie de Hodge, II, III, Publ.Math.IHES 40 (1971) 5-58; 44 (1974) 5-77.

[8] A. Dimca, Topics on Real and Complex Singularities, Vieweg Advanced Lectures in Math., 1987.

[9] A. Dimca, Singularities and Topology of Hypersurfaces, Universitext, Springer, 1992.

[10] A. Dimca, Sheaves in Topology, Universitext, Springer, 2004.

[11] A. Dimca and G.I. Lehrer, Purity and equivariant weight polynomials, in: Algebraic Groups and Lie Groups (ed. G.I. Lehrer), Cambridge University Press, 1997.

[12] A. Durfee and M. Saito, Mixed Hodge structures on the intersection cohomology of links, Compositio Math. 76 (1990), 49-76.

[13] J. Fogarty, Algebraic families on an algebraic surface, Amer. J. Math 90 (1968) 511-521.

[14] L. Göttsche, The Betti numbers of the Hilbert scheme of ponts on a smooth projective surface, Math. Ann. 286 (1990) 193-208.

[15] W. Greub, S. Halperin and R. Vanstone, Connections, curvature and cohomology, vol.III, Academic Press, 1976.

[16] P. Griffiths and J. Harris, Principles of Algebraic Geometry, Wiley, 1978.

[17] A. Iqbal, C. Kozçaz and C. Vafa, The refined topological vertex, arXiv:hep-th/0701156.

[18] S. Lang, Algebra, Addison-Wesley, 1965.

[19] M. E. Larsen, On the topology of complex projective manifolds, Invent. Math. 19 (1973), 251260.

[20] K. Lee, The singularities on the principal component of the Hilbert scheme of points, arXiv:0805.3672v2.

[21] S. Katz, The desingularization of $\mathrm{Hilb}^{4}\left(\mathbb{P}^{3}\right)$ and its Betti numbers, in: Zero-dimensional schemes (eds. F. Orecchia and L. Chiantini), de Gruyter, 1994.

[22] M. Kontsevich and Y. Soibelman, Stability structures, motivic Donaldson-Thomas invariants and cluster transformations, arXiv:0811.2435.

[23] D. Maulik, N. Nekrasov, A. Okounkov and R. Pandharipande, Gromov-Witten theory and Donaldson-Thomas theory, I, Comp. Math. 142 (2006), 1263-1285.

[24] H. Nakajima, Lectures on Hilbert schemes of points on surfaces, University Lecture Series 18, AMS, 1999.

[25] C. Peters and J. Steenbrink, Mixed Hodge Structures, Ergeb. der Math. und ihrer Grenz. 3. Folge 52, Springer, 2008.

[26] M. Saito, On microlocal b-function, Bull. Soc. Math. France 122 (1994), 163-184.

[27] M. Saito, Modules de Hodge polarisable, Publ. Res. Inst. Math. Sci. 24 (1988) 849-995.

[28] M. Saito, Mixed Hodge modules, Publ. Res. Inst. Math. Sci. 26 (1990) 221-333. 
[29] E. Segal, The $A_{\infty}$ deformation theory of a point and the derived categories of local Calabi-Yaus, J. Algebra 320 (2008) 3232-3268.

[30] J. Scherk and J. Steenbrink, On the mixed Hodge structure on the cohomology of the Milnor fiber, Math. Ann. 271, (1985), 641-665.

[31] B. Szendrői, Non-commutative Donaldson-Thomas invariants and the conifold, Geometry and Topology 12 (2008) 1171-1202.

[32] R. Thomas, A holomorphic Casson invariant for Calabi-Yau 3-folds and bundles on K3 fibrations, J. Diff. Geom. 54 (2000) 367-438.

Laboratoire J.A. Dieudonné, UMR du CNRS 6621, Université de Nice Sophia Antipolis, Parc Valrose, 06108 Nice Cedex 02, FRANCE.

E-mail address: dimca@unice.fr

Mathematical Institute, University of Oxford, 24-29 St Giles', Oxford, OX1 3LB, United KingDOM.

E-mail address: szendroi@maths.ox.ac.uk 